\title{
A retrospective cohort study of outcomes in hospitalised COVID-19 patients during the first pandemic wave in Ireland
}

\author{
Kenneth Beatty ${ }^{1}$ (D) Paul M. Kavanagh ${ }^{1,2}$ (1)
}

Received: 10 April 2021 / Accepted: 15 August 2021 / Published online: 19 November 2021

(c) Royal Academy of Medicine in Ireland 2021

\begin{abstract}
Aim Describe the epidemiology, resource use and adverse outcomes of COVID-19 patients hospitalised during wave 1 of the COVID-19 pandemic in Ireland. Use this data to identify specific cohorts at high risk of adverse outcomes and to inform acute hospital requirements for future COVID-19 waves in Ireland.

Methods The Health Service Executive's (HSE) Hospital Inpatient Enquiry (HIPE) system produced a daily database of COVID-19 discharge episodes from the onset of the COVID-19 pandemic in Ireland. This study analysed data on episodes of COVID-19 hospitalisation recorded between February 29 and July 31, 2020. A deterministic record linkage process transformed records from episode to patient level. Logistic regression modelling identified factors associated with long length of stay (LLOS), intensive care unit (ICU) admission and inhospital mortality.

Results Median length of stay was 9 days; $12.8 \%$ of patients had ICU admission and $16.6 \%$ died in hospital. Male patients were more likely to have ICU admission and die in hospital. Likelihood of LLOS and inhospital mortality increased with age. Obesity, hypertension and diabetes were associated with ICU admission while chronic kidney disease and chronic obstructive pulmonary disease were associated with inhospital mortality. Nursing home residents were less likely to be admitted to ICU and more likely to die in hospital compared to patients admitted from home.

Conclusion This study provides patient-level epidemiological characterisation of hospitalisations during the first COVID-19 pandemic wave in Ireland. The higher risk of adverse outcomes in older age groups supports the age-based prioritisation of COVID-19 vaccinations currently used in Ireland.
\end{abstract}

Keywords: COVID-19 · Intensive care unit · Inhospital mortality $\cdot$ Length of stay $\cdot$ Resource allocation

\section{Introduction}

In early 2020, severe acute respiratory syndrome coronavirus 2 (SARS-CoV-2) was linked to the emergence of a novel viral respiratory infection first reported in Wuhan, China [1, 2]. The typical symptoms and disease progression observed in this infection came to be known as coronavirus disease

Kenneth Beatty

kenneth.beatty@hse.ie

Paul M. Kavanagh

paul.kavanagh@hse.ie

1 Health Intelligence, Strategic Planning and Transformation, Jervis House, 4th FloorJervis Street, Dublin D01 W596, Ireland

2 Department of Epidemiology \& Public Health Medicine, Royal College of Surgeons in Ireland, Beaux Lane House, Mercer Street Lower, Dublin, Ireland
2019 or COVID-19 [2]. This disease has progressed into a pandemic affecting millions of people worldwide with the first case in Ireland notified on the 29th of February $2020[2,3]$. Following a suppression of the first wave of the COVID-19 pandemic, many countries, including Ireland, experienced a surge in cases in late 2020 [4-7], with identification of new SARS-CoV-2 variants and high numbers of admission to acute hospitals, particularly intensive care units (ICUs), reported. In order to ensure demand for acute hospital services does not exceed capacity in 2021, as well as ensuring sustainable delivery of non-COVID-19 care, contingency planning to maintain sufficiency of acute hospital services is a key element to health system response to the COVID-19 pandemic [8, 9].

In Ireland, baseline capacity challenges have existed in acute hospitals for many years. A recent report found Irish hospitals had approximately $30 \%$ fewer acute hospital beds than the Organisation for Economic Co-operation 
and Development (OECD) average [10]. A government review of health service capacity in Ireland in 2018 identified an immediate national level requirement of 1200 inpatient hospital beds and 60 adult critical care beds across all Irish hospitals to bring services to safe operating levels, $11 \%$ and $25 \%$ increases respectively [11]. The lack of ambulatory or inpatient pharmacological options to treat COVID-19 were key drivers for the introduction of non-pharmacological societal restrictions (known as lockdowns) to Ireland in mid-March 2020. However, the acute hospital bed capacity limitations highlighted here showed that Ireland was at high risk of demand for acute hospital services due to the COVID-19 pandemic outstripping supply, a narrative which was frequently published in the Irish media at the time [12, 13].

Median hospital lengths of stay (LOS) in hospitalised COVID-19 patients ranging from 4 to 21 days have been reported [14]. Pooled rate of ICU admission in COVID-19 patients was $26 \%$ (95\% confidence interval (CI) $20-33 \%$ ) with mortality rates of $31 \%$ (95\% CI 26-36\%) [15]. Presence of chronic obstructive pulmonary disease (COPD) (pooled (odds ratio) OR 17.8; 95\% CI 6.56-48.20), cardiovascular disease (pooled OR 4.4; 95\% 2.64-7.47) and hypertension (HTN) (pooled OR 3.7; 95\% CI 2.22-5.99) in COVID-19 patients were associated with ICU admission [16]. Risk factors associated with mortality in COVID-19 patients included HTN (OR 2.5; 95\% CI 2.07-3.09; $p<0.001$ ), coronary heart disease (OR $3.8 ; 95 \%$ CI $2.11-6.85 ; p<0.001)$ and diabetes (OR 2.0; $95 \%$ CI $1.67-2.31 ; p<0.001$ ) [17]. A retrospective study of COVID-19-positive patients summarised their study by stating "COVID-19 (patients) of older age, male gender, or having more than two comorbidities are at higher risk of hospitalization, mechanical ventilation and death" [18].

In the context of continuing surges in COVID-19 incidence early in the pandemic, and the ongoing threat to acute hospital resilience, the aim of this study was to characterise the epidemiology of COVID-19 hospitalised patients in wave 1 of the COVID-19 pandemic in Ireland and identify factors independently associated with adverse outcomes, specifically long length of stay, ICU admission and inhospital mortality. These data can be used to inform COVID-19-related health service demand and capacity modelling for future COVID-19 waves in Ireland, including enabling planning of health services to meet competing demand outside COVID-19. By identifying the much greater risk of adverse outcomes in hospitalised COVID-19 patients aged 65 years and older, it also provides local evidence which supports the National Immunisation Advisory Committee's (NIAC) decision to prioritise COVID-19 vaccine administration based on age cohort.

\section{Methods}

\section{Data source}

The Hospital In-Patient Enquiry (HIPE) system, maintained by the Healthcare Pricing Office (HPO), was the data source for this study [19]. This acute hospital discharge episode database is a well-established, quality-assured, national hospital care information system that uses the International Classification of Disease-10 -Australian Modification (ICD10-AM))/Australian Classification of Health Interventions (ACHI)/ Australian Coding Standards (ACS) coding to capture demographic, clinical and care data at discharge on all episodes of emergency and elective care across publicly funded hospitals in Ireland; it has been extensively used in epidemiological and health services research [20]. While there is private hospital activity in Ireland not captured in the system, in terms of inpatient care, that sector is focused almost exclusively on provision of elective care. Therefore, HIPE was likely to have high completeness for acute hospital episodes related to COVID-19 in Ireland. This study utilised interim HIPE records updated daily by the HPO to inform COVID-19 response planning. While later data validation exercises may have altered some of these records, this was the most accurate data on hospitalised cases of COVID-19 available at the time of the study.

\section{Inclusion criteria}

COVID-19-related discharge episodes in the HIPE file were defined by the presence of any of the following ICD10 codes across all diagnoses: U07.1, B34.2 or B97.2. All ICD-10AM/ACHI/ACS codes used to identify comorbidities and interventions in this report are listed in Table 1 . The $10^{\text {th }}$

Table 1 ICD-10-AM/ACHI/ACS Codes used to identify COVID-19 diagnosis, comorbidities and interventions analysed in this study

\begin{tabular}{ll}
\hline Comorbidities & ICD-10 Codes \\
\hline Obesity & E66.9 \\
Diabetes & E10.6 and E11.6 \\
Hypertension & I10 \\
COPD & J44.1-J44.9 \\
Asthma & J44 \& J45 \\
Chronic kidney disease & N18 \\
Influenza & J09, J10.0, J10.1, J10.8, J11.0, J11.1 \\
& or J11.8 \\
Interventions & ACHI Codes \\
Ventilation (all) & $1388200 / 1388201 / 1388202$ and \\
& $9220900 / 9220901 / 9220902)$ \\
Ventilation (invasive) & $1388200 / 1388201 / 1388202$ \\
Haemodialysis & $131000-131008$ \\
\hline
\end{tabular}


of August 2020 version of the daily interim HIPE record dataset, including discharge episodes up to and including that date and comprising 4837 records with admission dates from the $19^{\text {th }}$ of November 2018, was the primary data repository used in this study. In total, 189 records with admission dates prior to the date of Ireland's first confirmed case of COVID-19 (29 ${ }^{\text {th }}$ of February 2020) were excluded. These were presumed nosocomial infection, with hospital admission preceding onset of COVID-19 infection, and not new admissions arising from COVID-19 infection in the community, which was the study's focus. Records with an admission date between the $31^{\text {st }}$ of July and $10^{\text {th }}$ of August $2020(n=2)$ were excluded as they were likely to have much lower lengths of stay than the remainder of the dataset. This resulted in a dataset of COVID-19 discharges admitted from the 29th of February 2020 and discharged to the $31^{\text {st }}$ of July 2020, representing the first wave of the COVID-19 pandemic in Ireland, containing 4640 discharge episodes.

\section{Deterministic record linkage}

The HIPE database lacks national individual healthcare identifiers (IHIs). Discharge episodes where inter-hospital transfer or readmission occurs following a preceding episode for the same condition in the same patient are reported as new episodes rather than continuation of that patient's ongoing hospital care. This is especially problematic where inter-hospital transfer or readmission may be associated with adverse outcome and be a critical source of error. As the purpose of this study was to characterise hospitalised COVID19 patients and to identify risk factors for adverse outcomes, for epidemiological purposes, a patient-level rather than discharge episode-level analysis was required.

Firstly, 556 episodes where the admission source variable was recorded as an inter-hospital transfer were identified. These records were then internally linked with remaining records that matched on the gender, single year of age, and county of residence variables. Secondly, different forms of inpatient spells created by the linkage process were defined. In order to be defined as a Continuous Inpatient Spell (CIPS), episodes had to satisfy 5 matching criteria: admission source, age, gender, county of residence and discharge dates. When such records were created, the dates of admission, comorbidities, interventions and lengths of stay across the multiple episodes were merged. Where all 5 variables except for admission and discharge dates matched, readmission of the same patient was likely to have occurred. To ensure episodes referred to a single patient, Medical Record Numbers (MRNs) and hospitals of admission for the linked episodes had to match before merging occurred. This was defined as an Interrupted Inpatient Spell (IIPS). Records of discharge with matching MRNs and hospitals of admission with admissions from home were also identified as IIPSs. Cases where both inter-hospital transfer and readmission occurred were defined as Multiple Inpatient Spells (MIPS) and were managed in the same way as CIPS and IIPS. Finally, a number of records identified by HIPE as having an admission source as "Acute Hospital Transfer" had no preceding or subsequent episodes of admission. These records were termed Un-linkable Transfers (UT) and were included as individual patient episodes.

This deterministic record linkage process transformed 4640 hospital discharge episode-level records into 4086 patient-level records for the purpose of this study. To determine the completeness of the final database, it was compared to COVID-19 hospitalisations reported by the Health Protection Surveillance Centre (HPSC), which as of the $29^{\text {th }}$ of July 2020, numbered 3352 patients [21].

\section{Statistical analysis}

Adverse clinical outcomes analysed in this study were defined as "Long Length of Stay" (LLOS; any length of hospitalisation lasting greater than or equal to the upper quartile of all lengths of hospitalisation, i.e. 20 days), "Admission to ICU" and "In-Hospital Mortality", all of which are recorded as variables on the HIPE dataset. Frequency of key patient characteristics and stated adverse outcomes were measured overall and by age group.

Multivariable logistic regression models derived adjusted ORs (with 95\% CIs) measuring the strength of associations between independent variables and the outcomes of interest. Age group, gender and comorbidities were included in each model, then characteristics were selected based on a priori hypothesis of association informed by literature review and statistical significance (defined as $p<0.05$ ) on Chi-squared analysis [22]. Assumptions for logistic regression were reviewed and affirmed, including multicollinearity and influential outliers; goodness-of-fit was reviewed with Nagelkerke R Square statistics. All analyses were both performed using SPSS version 26 [23].

\section{Results}

\section{Overview of hospitalised COVID-19 patients}

Table 2 summarises the main characteristics of the hospitalised COVID-19 in the study. More male than female COVID-19 patients were admitted to hospital $(n=2226$; $54.5 \%$; 95\% CI 52.94-56.01\%), while the median age of patients was 64 years (Interquartile Range (IQR): 48-79 years). Most common comorbidities were diabetes $(n=741,18.1 \%$; 95\% CI $16.96-19.35 \%)$, COPD $(n=338$; 8.3\%; 95\% CI 7.45-9.16\%) and chronic kidney disease (CKD) $(n=319 ; 7.8 \%$; 95\% CI 7.00-8.67\%). 
Table 2 Characteristics of hospitalised COVID-19 cases admitted to hospitals in Ireland from February 29, 2020, and discharged by July 31,2020 , stratified by patient level

\begin{tabular}{|c|c|c|}
\hline \multirow[t]{2}{*}{ Characteristics } & \multicolumn{2}{|c|}{$\begin{array}{l}\text { Total number of hospitalised } \\
\text { cases }\end{array}$} \\
\hline & $n(\%)$ & $95 \% \mathrm{CI}$ \\
\hline Total & $4086(100.0)$ & \\
\hline \multicolumn{3}{|l|}{ Sex } \\
\hline Male & $2226(54.5)$ & $(52.94-56.01)$ \\
\hline Female & $1860(45.5)$ & $(43.99-47.06)$ \\
\hline \multicolumn{3}{|l|}{ Age in years } \\
\hline Median (IQR) & $64(48-79)$ & \\
\hline \multicolumn{3}{|l|}{ Age groups (years) } \\
\hline 0-39 & $605(14.8)$ & $(13.73-15.93)$ \\
\hline $40-64$ & $1440(35.2)$ & (33.78-36.73) \\
\hline $65-84$ & $1511(37.0)$ & $(35.50-38.48)$ \\
\hline $85+$ & $530(13.0)$ & (11.96-14.04) \\
\hline \multicolumn{3}{|l|}{ Comorbidities } \\
\hline Obesity & $96(2.3)$ & $(1.91-2.86)$ \\
\hline Diabetes & $741(18.1)$ & $(16.96-19.35)$ \\
\hline HTN & $303(7.4)$ & $(6.63-8.26)$ \\
\hline COPD & $338(8.3)$ & $(7.45-9.16)$ \\
\hline Asthma & $132(3.2)$ & $(2.71-3.82)$ \\
\hline CKD & $319(7.8)$ & $(7.00-8.67)$ \\
\hline Influenza & $75(1.8)$ & $(1.45-2.30)$ \\
\hline \multicolumn{3}{|l|}{ Multiple comorbidities } \\
\hline $\mathbf{0}$ & $2556(62.6)$ & $(61.05-64.04)$ \\
\hline 1 & $1134(27.8)$ & $(26.38-29.15)$ \\
\hline 2 & $322(7.8)$ & $(7.07-8.75)$ \\
\hline 3 & $70(1.7)$ & $(1.34-2.16)$ \\
\hline 4 & $<5(0.1)$ & $(0.03-0.25)$ \\
\hline \multicolumn{3}{|l|}{ Admission source } \\
\hline Home & $3413(83.5)$ & $(82.36-84.65)$ \\
\hline Nursing home & $387(9.5)$ & $(8.59-10.41)$ \\
\hline Transfer from acute hospital & $260(6.4)$ & $(5.63-7.16)$ \\
\hline Other & $26(0.6)$ & $(0.42-0.93)$ \\
\hline \multicolumn{3}{|l|}{ Adverse outcomes } \\
\hline Long length of stay & $1072(26.2)$ & $(24.89-27.61)$ \\
\hline ICU admission & $521(12.8)$ & $(11.74-13.81)$ \\
\hline Inhospital mortality & $677(16.6)$ & $(15.44-17.74)$ \\
\hline
\end{tabular}

\section{Occurrence of adverse outcomes}

Table 3 summarises occurrences of adverse outcomes stratified by age group. Median LOS was 9 days with an interquartile range of 4 to 20 days. In total, $12.8 \%(n=521)$ of COVID-19 patients admitted to hospital required ICU admission and $16.6 \%(n=677)$ died in hospital. A high proportion of patients experiencing LLOS $(37.9 \%$; $95 \%$ CI $33.78-42.21 \%)$ and in-hospital mortality $(41.9 \%$; $95 \%$ CI $37.65-46.22 \%$ ) was observed in the 85 years and older age group. Patients aged 40-64 years had the highest proportion of ICU admission (18.8\%; 95\% CI 16.76-20.86\%).

\section{Risk factors for adverse outcomes}

Table 4 displays independent associations between COVID19 patient characteristics and adverse clinical outcomes derived from logistic regression modelling. Overall, models displayed Nagelkerke R Square statistic results between 19.2 and $31.1 \%$.

Patients' gender showed no statistically significant association with LLOS but odds of LLOS in hospitalised COVID-19 did increase with age. Hospitalised COVID-19 patients aged over 85 years were over 11 times more likely than those aged $0-39$ years to have LLOS (aOR 11.2; 95\%CI 7.39-17.07; $p<0.001)$. Male patients were 53\% more likely than females (aOR 1.5; 95\% CI 1.25-1.88; $p<0.001$ ) and patients over 85 years were $66 \%$ less likely than those aged under 40 years (aOR 0.3 ; 95\%CI $0.18-0.63 ; p=0.001$ ) to be admitted to ICU. Male patients were over $20 \%$ more likely than female patients to die in hospital (aOR 1.2; 95\% CI $1.02-1.49 ; p=0.032$ ), while the likelihood of in-hospital mortality increased with age.

Long length of stay in COVID-19 patients was associated with obesity (aOR 3.4; 95\% CI 2.12-5.29; $p<0.001$ ), diabetes (aOR 1.3, 95\% CI 1.09-1.55; $p=0.01$ ), HTN (aOR 2.8; 95\% CI 2.15-3.58; $p<0.001)$ and CKD (aOR 1.4; 95\%CI $1.05-1.76 ; p=0.021)$ respectively. Obesity (aOR 7.9; 95\% CI 5.42-12.45, $p<0.001$ ), diabetes (aOR 1.3, 95\%CI $1.01-1.66 ; p=0.039)$ and HTN (aOR 4.3; 95\% CI 3.21-5.68, $p<0.001)$ also had strong association with ICU admission amongst hospitalised COVID-19 patients. COPD (aOR 1.5; 95\% CI 1.16-2.01; $p=0.002)$ and CKD (aOR 1.9; 95\% CI $1.49-2.64 ; p<0.001)$ were the only comorbidities to show statistically significant associations with inhospital mortality. Of note, co-infection with COVID-19 and influenza did not show statistically significant independent associations with LLOS, ICU admission or inhospital mortality. The number of comorbidities per patient did not retain significance or add to $\mathrm{R}$ squared value in the final models.

COVID-19 patients admitted from nursing homes were $85 \%$ less likely to be admitted to ICU (aOR 0.2; 95\% CI $0.07-0.33 ; p<0.001)$ but over twice as likely to die in hospital (aOR 2.3; 95\% CI 1.74-2.90; $p<0.001$ ) compared to patients admitted from home. Patients transferred from another hospital had higher odds of LLOS (aOR 3.8; 95\% CI $2.19-6.46 ; p<0.001)$ and ICU admission $(1.6 ; 95 \% \mathrm{CI}$ $1.04-2.51 ; p=0.034)$ with escalation of care showing a greater than twofold likelihood of ICU admission (aOR 2.4; 1.22-4.54; $p=0.011$ ).

Finally, patients admitted in March or April were more likely to require LLOS (aOR 1.9; 95\%CI 1.57-2.52: $p<0.001$ ) be admitted to ICU (aOR 2.28; 95\% CI 1.59-3.26; 
Table 3 Overview of the occurrence of adverse outcomes stratified by age group

\begin{tabular}{|c|c|c|c|c|}
\hline \multirow[b]{2}{*}{$\begin{array}{l}\text { Age group } \\
\text { (years) }\end{array}$} & \multirow[b]{2}{*}{$n(\%)$} & \multicolumn{3}{|c|}{ Adverse outcomes } \\
\hline & & $\begin{array}{l}\text { Long LOS } \\
\%(95 \% \mathrm{CI})\end{array}$ & $\begin{array}{l}\text { ICU admission } \\
\%(95 \% \mathrm{CI})\end{array}$ & $\begin{array}{l}\text { Inhospital mortality } \\
\%(\mathbf{9 5} \% \mathrm{CI})\end{array}$ \\
\hline $0-39$ & $605(14.8)$ & $\begin{array}{r}5.3 \\
(3.65-7.39)\end{array}$ & $\begin{array}{r}8.1 \\
(6.05-10.57)\end{array}$ & $\begin{array}{r}1.3 \\
(0.57-2.59)\end{array}$ \\
\hline $40-64$ & $1440(35.2)$ & $\begin{array}{c}20.2 \\
(18.16-22.38)\end{array}$ & $\begin{array}{c}18.8 \\
(16.76-20.86)\end{array}$ & $\begin{array}{r}5.0 \\
(3.93-6.26)\end{array}$ \\
\hline $65-84$ & $1511(38.0)$ & $\begin{array}{c}35.3 \\
(32.95-37.77)\end{array}$ & $\begin{array}{c}12.1 \\
(10.54-13.85)\end{array}$ & $\begin{array}{c}24.8 \\
(22.66-27.08)\end{array}$ \\
\hline $85+$ & $530(13.0)$ & $\begin{array}{c}37.9 \\
(33.78-42.21)\end{array}$ & $\begin{array}{r}2.6 \\
(1.45-4.39)\end{array}$ & $\begin{array}{c}41.9 \\
(37.65-46.22)\end{array}$ \\
\hline Total & $4086(100.0)$ & $\begin{array}{c}26.2 \\
(24.89-27.61)\end{array}$ & $\begin{array}{c}12.8 \\
(11.74-13.81)\end{array}$ & $\begin{array}{c}16.6 \\
(15.44-17.74)\end{array}$ \\
\hline
\end{tabular}

$p<0.001$ ) and die inhospital (aOR 1.9; 95\% CI 1.51-2.64; $p<0.001$ ) than patients admitted in May, June or July. Patients admitted to ICU were almost 3 times more likely to die inhospital (aOR 2.8; 95\%CI 2.06-3.66: $p<0.001$ ).

\section{Discussion}

This study has characterised patients hospitalised with COVID-19 during the first wave of pandemic 2020 in Ireland and has identified risk factors for adverse outcomes of longer length of stay, ICU admission and inhospital mortality by using a quality-assured national dataset, transformed by deterministic record linkage for epidemiological analysis. These findings can inform hospital preparedness for future surges in COVID-19 incidence and overall pandemic response planning as well as local epidemiological evidence in support of the COVID-19 Vaccine Implementation Plan [24],

\section{Key findings and comparisons with other studies}

\section{Age and gender}

This study showed that male COVID-19 patients admitted to hospital were more likely to require ICU admission and to die inhospital than their female counterparts, a finding supported by previously published studies [25, 26]. Male COVID-19 patients, particularly elderly male patients, have been identified by this study as being at especially high risk of adverse outcomes once admitted to hospital. COVID-19 information strategies and vaccine uptake drives targeting this cohort should be considered in preparation for any potential future waves.

This study found increasing age was independently associated with a greater likelihood of adverse outcomes, except ICU admission. Similar to previously published studies, the likelihood of mortality amongst COVID-19 inpatients increased with age $[17,27]$. This risk peaked in patients aged 85 years and older who were over 57 times as likely as those aged 40 years and younger to die in hospital. While older age cohorts are frequently associated with poorer medical outcomes in general, the extremely large increase in the risk of inhospital mortality, in particular, in older age groups compared to their younger counterparts highlights the importance of exhausting all available resources to protect this vulnerable section of our society from COVID-19. Although associations between age and ICU admission raise questions about challenging clinical decision during a pandemic on prioritisation for scarce resources, full adjustment for the wide range of factors that influence such a significant step in clinical management would be required to explore this further, and was greatest in the 85 and older age group.

In Ireland, like many other countries, early in the COVID19 pandemic, specific protective advice, referred to as "cocooning", was recommended for older people in Ireland because of concern they were at high risk of poor outcomes [28]. Ireland has recently been able to remove most nonpharmacological interventions (NPIs), due in no small part to the high level of vaccine uptake in high-risk cohorts. However, the emergence of variants of SARS-CoV-2 and their ability to escape the effect of vaccines mean the reintroduction of NPIs may be required to control potential surges of COVID-19 in autumn/winter 2021. While concerns about the potential harms of this protective measure have been raised [29], this study confirms the importance of public health measures such as cocooning to protect the most vulnerable members of our society and can provide local empirical evidence of the necessity of NPIs should they once again be required.

\section{Comorbidities}

Similar to findings reported in existing literature, obesity, hypertension and diabetes were associated with both LLOS and ICU admission, with CKD also associated with LLOS 


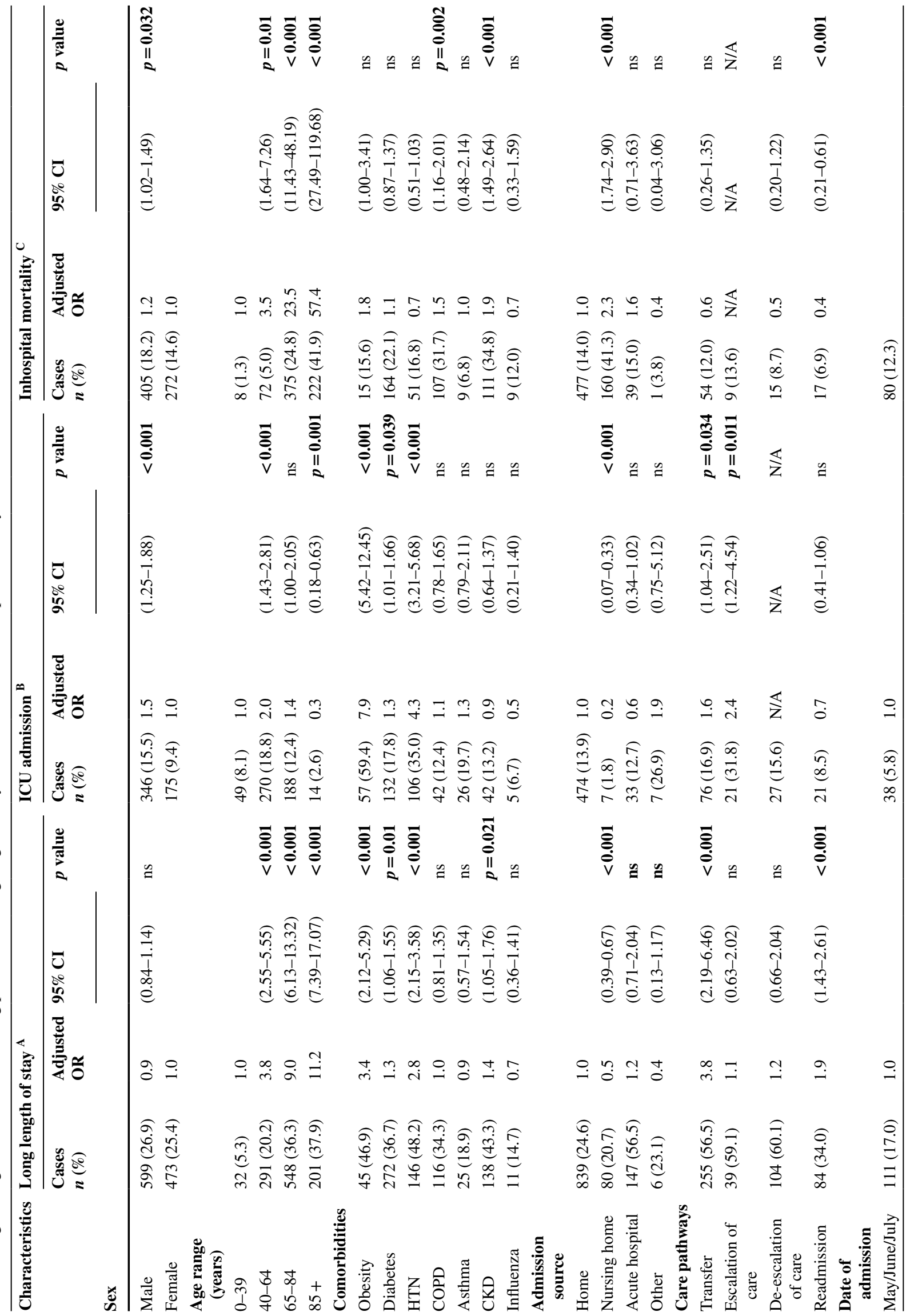




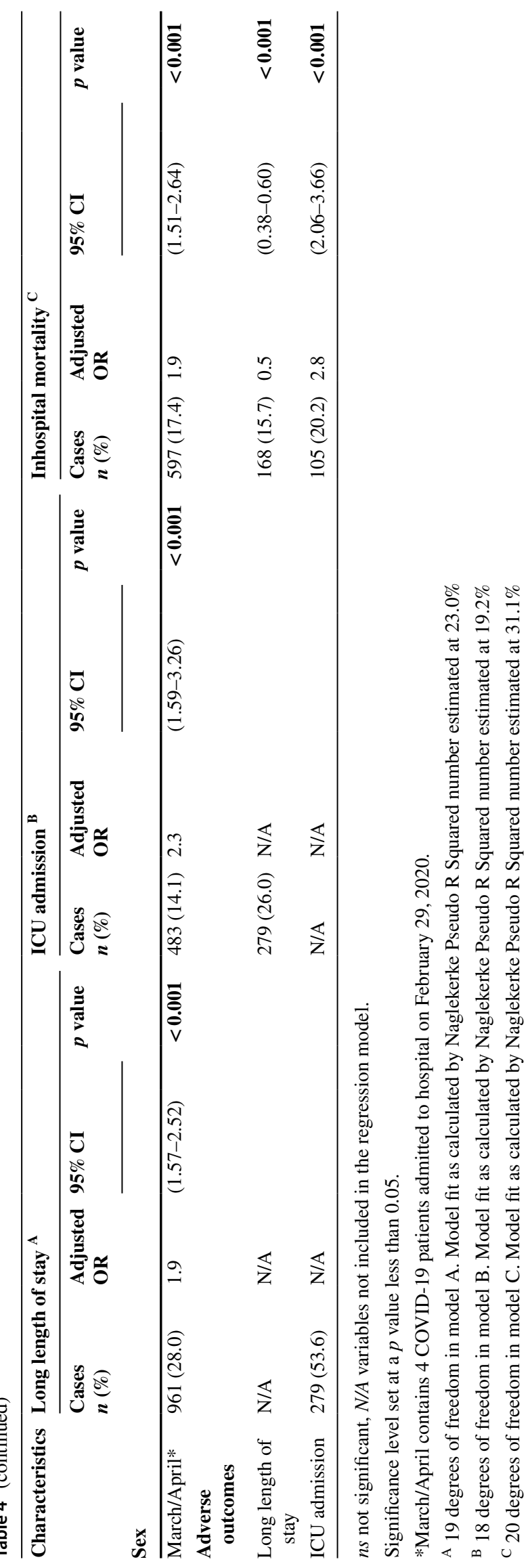


$[14,30]$. This association of highly prevalent comorbidities like obesity, hypertension, diabetes and CKD with LLOS and ICU admission shows that hospital resources can very rapidly be put under significant strain depending on the casemix of patients admitted to hospital.

Identification of obesity in this study relied on it being recorded in discharge paperwork, a limitation of HIPE data previously reported in medical literature [31]. Allowing for this limitation, obesity has shown to be associated with ICU admission in COVID-19 patients and this study describes a similar yet stronger association amongst Irish hospitalised COVID-19 patients [32]. The Healthy Ireland survey reported 23\% of adults in Ireland were obese [33], a common public health challenge in most countries. The findings from this and similar studies regarding the relationship between obesity and severe COVID-19 disease progress highlight the importance of health systems taking a wide view on population risk management during the pandemic. Recommendations from "A Healthy Weight for Ireland- Obesity policy and action plan" should be examined to identify opportunities provided by COVID-19 to improve physical activity and obesity rates in Ireland [34].

Non-communicable diseases (NCDs) in general increase the risk of severe COVID-19, with the World Health Organization (WHO) underlining the importance of implementing policies to "treat people living with NCDs and prevent and control their risk factors during the COVID-19 outbreak" $[35,36]$. Primary and secondary prevention policies included in the "National Framework for the Integrated Prevention and Management of Chronic Disease in Ireland 2020-2025" have potential to reduce the impact of NCDs on general health and help mitigate the impact of COVID19 [37].

An important negative finding was that co-infection with influenza in COVID-19 patients was not associated with any adverse outcomes measured, contrary to results of previous studies [38]. Numbers of influenza infection in this report $(n=75 ; 1.8 \%)$ were quite low, possibly representative of the period of the report where circulating influenza was at low levels [39].

\section{Patient pathways}

Independent of age and comorbidity, COVID-19 patients admitted from nursing homes were over twice as likely to die in hospital as those admitted from home. Lack of adjustment for frailty and dementia may also explain this relationship [40]. International studies have highlighted the increased mortality rates from COVID-19 in nursing homes, but this is the first Irish study that compares hospitalised COVID-19 patients admitted from nursing homes with those admitted from private homes [41]. The impact of COVID-19 on nursing home residents in Ireland and weaknesses in the sector which contributed to the issue were heavily criticised, leading to a special national review of the issue. The COVID-19 Nursing Home Expert Panel outlined key recommendations to mitigate future outbreaks of COVID-19 in these facilities, and the findings in this study add weight to the urgency of implementation [42]. It also provides empirical evidence supporting the decision to give early access to COVID-19 vaccines to nursing home residents as one of the highest priority groups in vaccine roll-out in Ireland [24].

\section{Strengths and weaknesses}

This is the first study that reports on hospitalised COVID19 patients in Ireland at a patient level. It used a national quality-assured dataset and includes a large number of hospitalised COVID-19 patients which enables the characterisation and identification of predictors of adverse clinical outcomes with precision. Secondary data analysis is a well-established and efficient approach to epidemiological research, and this study benefited from the advantages of a database with high completeness for the COVID-19 hospitalised patients [21], a robust and externally reviewed data quality management system and a large sample size. While the HIPE database did not report on COVID-19 patients admitted to Irish private hospitals, in general, COVID-19 patients with acute care requirements were admitted to HSE hospitals so this data source is likely to be complete for this study's patients of interest. The risk of data-dredging was minimised through careful a priori selection of risk factors for analysis. A particular strength of the study is deterministic record linkage which produced a patient-level database from an episode-level database. This meant the associations between characteristics and adverse outcomes quoted in this study are as specific to individual patients as possible.

There were some limitations to this study. Although HIPE is a well-recognised and widely used repository of data on patients discharged from hospitals in Ireland, this study relied on secondary data analysis of variables not collected for the purpose of the research question. This study analysed COVID-19 patients admitted to hospital with limited use of subgroup analyses. This form of analysis was chosen to provide an overview of the demographics and adverse outcomes associated with all hospitalised COVID-19 inpatients early in the pandemic to inform pandemic planning for future pandemic waves. Some data (e.g. comorbidities) may be incomplete and that incompleteness was possibly associated with outcomes studied. In the absence of IHIs, the deterministic record linkage process may have resulted in under- or over-matching of patients; however, while no gold standard exists to confirm completeness, agreement in enumeration of hospitalised patients was confirmed by comparison with the national 
infectious disease reporting system in Ireland [21]. Mortality described in this report was limited to hospitalised COVID-19 patients which may underestimate the overall mortality of COVID-19 cases in wave 1 of the pandemic in Ireland. Despite the above limitations, the HIPE database was the most accurate and up to date repository of data on hospitalised cases in Ireland at the time of the study and as such was the best available data source to inform future HSE policy relating to COVID-19 inpatients.

The regression models in this report had model fits ranging from 19.2 to $31.1 \%$ and do not fully explain the association between the independent variables and the outcomes of interest. The variables available to build the models were constrained by the variables available in the source dataset, which was subject to secondary analysis. Factors external to what was possible to examine in this study may have influenced these outcomes. For example, high numbers of COVID-19 outbreaks in nursing homes during the study's timeframe may have affected patient egress, ultimately resulting in longer lengths of stay or patients from areas of high social deprivation having higher rates of mortality, which was reported in other jurisdictions [43]. There is an opportunity to explore further prognostic indicators in future studies; however, this study does provide a valuable insight into outcomes for COVID-19 inpatients during wave 1 in Ireland and is robust enough to support policies such as the COVID-19 Vaccine Implementation Plan [24].

\section{Summary and conclusion}

Until population immunity reaches sufficient levels to control transmission, the current policy of NPI relaxation and the emergence of SARS-Co-2 variants of concern may result in future surges of the COVID-19 pandemic. Therefore, it is crucial that during periods of high COVID-19 incidence, health service preparedness is informed by characteristics that identify patients at higher risk of prolonged hospitalisation, of requiring critical care and of inhospital mortality. This study identified these characteristics which can now be used to support pandemic preparedness for future COVID-19 surges. Findings of this report provide a strong, local empiric basis to support existing public health policies introduced to protect vulnerable populations, such as the programme of age-based prioritisation for COVID-19 vaccine administration advocated by NIAC. While international studies are helpful, local data is necessary to tailor data analysis and modelling to inform local responses; use of local data also builds the confidence of decision-makers in using evidence for planning and management [44]. The COVID-19 vaccine administration programme has progressed rapidly in this country, giving us reasons to hope for a future without the need for NPIs. However, should they be required again in the future, this study underlines the crucial role they play in protecting society's most vulnerable.

\section{Key points}

- Increasing age was independently associated with greater likelihood of adverse outcomes, except ICU admission.

- The likelihood of inhospital mortality increased with age and was greatest in the 85 and older age group.

- Independent of age and comorbidity COVID-19 patients admitted from nursing homes were over twice as likely to die in hospital as those admitted from home.

- Obesity, hypertension and diabetes were associated with both long length of stay (LLOS), intensive care unit (ICU) admission, with chronic kidney disease also associated with LLOS

- Patients admitted in March or April were more likely to require LLOS, be admitted to ICU and die inhospital than patients admitted in May, June or July.

Acknowledgements This study used interim HIPE data from the first wave of the of the COVID-19 pandemic in Ireland which was specially prepared by the Healthcare Pricing Office to enable planning and response. We are grateful to Dr Howard Johnson, Clinical Lead Health Intelligence, Health Service Executive (HSE), who enabled access to HIPE data via the Health Atlas Ireland health analytics platform. We are also grateful to Dr Siobhan Ní Bhriain, Lead for Integrated Care, HSE, and Dr Colm Henry, Chief Clinical Officer, HSE, who provided helpful comments on draft versions of the work presented in this article.

Availability of data and material The Hospital In-Patient Enquiry (HIPE) system, maintained by the Healthcare Pricing Office (HPO), was the data source for this study. This study utilised interim HIPE records updated on a daily basis by the HPO to inform COVID-19 response planning.

Code availability Not applicable.

\section{Declarations}

Ethics approval Ethical review was not required for this study. The data used in the study are controlled by the HSE in Ireland. The study authors are registered medical professionals. Legal duties, organisational policies and good practices were observed in data handling and the data processing for the study was conducted for medical purposes by the authors to inform the statutory function of the HSE in Ireland to improve, promote and protect the health and welfare of the public (Sect. 7, Health Act 2004), thus consistent with General Data Protection Regulations and their application in Ireland. HIPE data is anonymised for users and usual practices regarding statistical disclosure control were applied.

Competing interests The authors declare no competing interests.

Consent to participate Not applicable.

Consent for publication Not applicable. 


\section{References}

1. World Health Organization (2020) Timeline: WHO's COVID-19 response. Available from: https://www.who.int/ emergencies/diseases/novel-coronavirus-2019/interactivetimeline. Accessed 19 September 2020

2. Zheng J (2020) SARS-CoV-2: an Emerging coronavirus that causes a global threat. Int J Biol Sci 16(10):1678-1685. https:// doi.org/10.7150/ijbs.45053

3. O'Brien F (2020) First case of COVID-19 diagnosed in east of Ireland. Radio Teleifis Eireann (RTE) News. https://www.rte. ie/news/coronavirus/2020/0229/1119357-coronavirus-ireland/ Accessed 19 September 2020

4. Remuzzi A, Remuzzi G (2020) COVID-19 and Italy: what next? Lancet 395(10231):1225-1228. https://doi.org/10.1016/S01406736(20)30627-9

5. Montesó-Curto P, Sánchez-Montesó L, Maramao FS, Toussaint L (2020) Coping with the COVID-19 pandemic in Italy and Spain: lessons in response urgency. J Glob Health 10(2):020326. https://doi.org/10.7189/jogh.10.020326

6. Carroll WD, Strenger V, Eber E et al (2020) European and United Kingdom COVID-19 pandemic experience: the same but different. Paediatr Respir Rev 35:50-56. https://doi.org/10. 1016/j.prrv.2020.06.012

7. European Centre for Disease Prevention and Control (ECDC) Risk related to spread of new SARSCoV-2 variants of concern in the EU/EEA. https://www.ecdc.europa.eu/sites/default/files/ documents/COVID-19-risk-related-to-spread-of-new-SARSCoV-2-variants-EU-EEA.pdf. Accessed 3 February 2021

8. Christen P, D'Aeth JC, Løchen A, McCabe R, Rizmie D, Schmit N, et al (2021) The J-IDEA Pandemic Planner. Med Care 59(5):371378. https://doi.org/10.1097/MLR.0000000000001502

9. European Centre for Disease Prevention and Control (ECDC) https://www.ecdc.europa.eu/en/publications-data/guidancehealth-system-contingency-planning-during-wides preadtransmission-sars. Accessed 5 January 2021

10. Keegan C, Brick A, Walsh B et al (2019) How many beds? Capacity implications of hospital care demand projections in the Irish hospital system, 2015-2030. Int J Health Plann Manage 34(1):e569-e582

11. Department of Health. Health Service Capacity Review (2018) Review of health demand and capacity requirements in Ireland to 2031. https:// assets.gov.ie/10132/7c2a2299ca924852b3002e9700253bd9.pdf. Accessed February 42021

12. Clarke V (2020) Covid-19: Lockdown needed to protect health service, say medics. Irish Examiner. https://www.irishexaminer. com/news/arid-40059557.html Accessed March 52021

13. Radio Teilifis Eireann (RTE) News. Timeline: Six months of Covid-19. https://www.rte.ie/news/newslens/2020/0701/ 1150824-coronavirus/. Accessed 20 November 2020

14. Rees EM, Nightingale ES, Jafari Y et al (2020) COVID-19 length of hospital stay: a systematic review and data synthesis. BMC Med 18:270. https://doi.org/10.1186/s12916-020-01726-3

15. Abate SM, Ahmed Ali S, Mantfardo B et al (2020) Rate of Intensive Care Unit admission and outcomes among patients with coronavirus: A systematic review and Meta-analysis. PLoS One 15(7):e0235653. https://doi.org/10.1371/journal.pone. 0235653

16. Jain V, Yuan JM (2020) Predictive symptoms and comorbidities for severe COVID-19 and intensive care unit admission: a systematic review and meta-analysis. Int J Public Health 65(5):533-546. https://doi.org/10.1007/s00038-020-01390-7

17. Tian W, Jiang W, Yao J et al (2020) Predictors of mortality in hospitalized COVID-19 patients: a systematic review and meta-analysis. J Med Virol 92(10):1875-1883. https://doi.org/ 10.1002/jmv. 26050

18. Gerwen M, Alsen M, Little C et al (2021) Risk factors and outcomes of COVID-19 in New York City; a retrospective cohort study. J Med Virol [Internet] 93(2):907-915. https://doi.org/10. 1002/jmv.26337

19. Healthcare Pricing Office. Hospital in patient enquiry instruction manual. http://www.hpo.ie/hipe/hipe_instruction_manual/ HIPE_Instruction_Manual_1.1.2016.pdf. Accessed 11 November 2020

20. Moloney ED, Smith D, Bennett K et al (2005) Do consultants differ? Inferences drawn from hospital in-patient enquiry (HIPE) discharge coding at an Irish teaching hospital. Postgrad Med J 81(955):327-332. https://doi.org/10.1136/pgmj.2004. 026245.

21. Health Protection Surveillance Centre. Epidemiology of COVID-19 in Ireland. https://www.hpsc.ie/a-z/respiratory/ coronavirus/novel coron avirus/casesinire land/ epidemiologyofcovid-19inireland/july2020/COVID-19. Daily_epidemiology_report_(NPHET)_31072020\%20-\% 20Website.pdf. Accessed 2 March 2021

22. Interpretation and Uses of Medical Statistics, 5th Edition. Leslie Daly, Geoffrey J Bourke. ISBN: 978-0-632-04763-5 August 2000 Wiley-Blackwell

23. IBM SPSS Statistics for Windows, Version 27.0. Armonk, NY: IBM Corp

24. Government of Ireland. COVID-19 vaccine strategy and implementation plan. Available at: https://www.gov.ie/en/publication/ bf337-covid-19-vaccination-strategy-and-implementation-plan/ Accessed 9 February 2021

25. Grasselli G, Zangrillo A, Zanella A et al (2020) Baseline Characteristics and Outcomes of 591 Patients Infected With SARSCoV-2 Admitted to ICUs of the Lombardy Region, Italy. JAMA 323(16):1574-81. https://doi.org/10.1001/jama.2020.5394

26. Lewnard JA, Liu VX, Jackson ML et al (2020) Incidence, clinical outcomes, and transmission dynamics of severe coronavirus disease 2019 in California and Washington: prospective cohort study. BMJ 369:1923. https://doi.org/10.1136/bmj.m1923

27. Petrilli CM, Jones SA, Yang J et al (2020) Factors associated with hospital admission and critical illness among 5279 people with coronavirus disease 2019 in New York City: prospective cohort study. BMJ 369(1966). https://doi.org/10.1136/bmj. m1966

28. Health Protection Surveillance Centre. Guidance on cocooning to protect people over 70 years and those extremely medically vulnerable from COVID-19 v1.5. https://www.lenus.ie/handle/10147/ 627623. Accessed 9 January 2021

29. The Irish Longitudinal Study on Ageing (TILDA) Altered lives in a time of crisis: the impact of the COVID-19 pandemic on the lives of older adults in Ireland. https://tilda.tcd.ie/publications/ reports/C19ReportKeyFindings/index.php. Accessed 9 February 2021

30. Moriconi D, Masi S, Rebelos E et al (2020) Obesity prolongs the hospital stay in patients affected by COVID-19, and may impact on SARS-COV-2 shedding. Obes Res Clin Pract 14(3):205209. https://doi.org/10.1016/j.orcp.2020.05.009

31. Vellinga A, O'Donovan D, De La Harpe D (2008) Length of stay and associated costs of obesity related hospital admissions in Ireland. BMC Health Serv Res 8:88. https://doi.org/10.1186/ 1472-6963-8-88

32. Kalligeros M, Shehadeh F, Mylona EK et al (2020) Association of obesity with disease severity among patients with coronavirus disease 2019. Obesity 28(7):1200-1204. https://doi.org/10.1002/ oby. 22859 
33. Government of Ireland. Healthy Ireland Survey documents. https:// www.gov.ie/en/collection/231c02-healthy-ireland-survey-wave/ Accessed 5 February 2021

34. Government of Ireland. A healthy weight for Ireland. Obesity policy and action plan. https://www.gov.ie/en/publication/c778a9-ahealthy-weight-for-ireland-obesity-policy-and-action-plan-2016202/. Accessed 9 February 2021

35. Clark A, Jit M, Warren-Gash $\mathrm{C}$ et al Global, regional, and national estimates of the population at increased risk of severe COVID-19 due to underlying health conditions in 2020: a modelling study. Lancet Global Health 8(8):1003-1017. https://doi.org/10.1016/ S2214-109X(20)30264-3

36. World Health Organization. The impact of the COVID-19 pandemic on noncommunicable disease resources and services: results of a rapid assessment. https://www.who.int/publicationsdetail-redirect/9789240010291. Accessed 9 February 2021

37. Health Service Executive. National framework for the integrated prevention and management of chronic disease in Ireland 2020-2025. https://www.hse.ie/eng/about/who/cspd/icp/chronicdisease/documents/national-framework-integrated-care.pdf. Accessed 12 December 2020

38. Stowe J, Tessier E, Zhao H et al (2021) Interactions between SARS-CoV-2 and influenza, and the impact of coinfection on disease severity: a test-negative design. Int J Epidemiol 50(4):11241133.https://doi.org/10.1093/ije/dyab081

39. Health Protection Surveillance Centre. Influenza Surveillance in Ireland - Weekly Report; Influenza Week 162020 (13th-19th April 2020). https://www.hpsc.ie/a-z/respiratory/influenza/ seasonalinfluenza/surveillance/influenzasurveillancereports/ previousinfluenzaseasonssurveillancereports/20192020season/ Influenza_Surveillance_Report_Week\%2016.pdf. Accessed 21 September 2020

40. Theou O, Squires E, Mallery K et al (2018) What do we know about frailty in the acute care setting? A scoping review BMC Geriatr 18:139. https://doi.org/10.1186/s12877-018-0823-2

41. Fallon A, Dukelow T, Kennelly SP, O'Neill D (2020) COVID-19 in nursing homes. QJM 113(6):391-392. https://doi.org/10.1093/ qjmed/hcaa136

42. Government of Ireland. COVID-19 Nursing Homes Expert Panel: Final Report. https://www.gov.ie/en/publication/3af5a-covid-19nursing-homes-expert-panel-final-report/. Accessed 9 December 2020

43. Woodward M, Peters SAE, Harris K (2021) Social deprivation as a risk factor for COVID-19 mortality among women and men in the UK Biobank: nature of risk and context suggests that social interventions are essential to mitigate the effects of future pandemics. J Epidemiol Community Health. 75(11):1050-1055. https://doi. org/10.1136/jech-2020-215810

44. van de Goor I, Hämäläinen RM, Syed A et al (2017) Determinants of evidence use in public health policy making: results from a study across six EU countries. Health Policy 121(3):273-281. https://doi.org/10.1016/j.healthpol.2017.01.003

Publisher's Note Springer Nature remains neutral with regard to jurisdictional claims in published maps and institutional affiliations. 